\title{
GLOBALIZZAZIONE, MOBILITÀ UMANA E CREATIVITÀ: RIVISITANDO CATEGORIE A PARTIRE DA TRE CASI DI MIGRAZIONE FORZATA IN ANGOLA
}

\author{
Globalization, human mobility and creativity: \\ revisiting categories from three cases of forced migration in Angola
}

Paulo Inglês*

\begin{abstract}
Riassunto. Questo articolo raccoglie alcune riflessioni sull'esperienza di ricerca in Angola nell'ambito della mobilità umana, con l'obiettivo di riflettere sulla produzione di classificazioni e categorie da parte di agenzie internazionali, ONG, policy-makers e ricercatori. Dopo aver sottolineato i rischi dell'assunzione acritica in attività di ricerca accademica di classificazioni e categorie prodotte in ambito burocratico, l'autore propone $\mathrm{d}^{\prime}$ interpretare la migrazione come un'azione creativa alla quale individui o gruppi umani ricorrono per affrontare circostanze avverse e ricostruire la quotidianità.
\end{abstract}

Parole chiave: migrazioni; classificazioni; categorie; creatività; Angola.

\begin{abstract}
This article enumerates some of the experiences of research in Angola in the field of hu man mobility, aiming to reflect on the production of classifications and categories by international agencies, NGOs, policy makers and researchers. After stressing the risks of uncritical use in academic research activities of classifications and categories produced in the bureaucratic field, the author proposes to interpret migration as a creative action to which individuals or human groups resort to facing adverse circumstances and reconstructing everyday life.
\end{abstract}

Keywords: migrations; classifications; categories; creativity; Angola.

\footnotetext{
* Università Bundeswehr. Monaco, Germania.
} 


\section{Introduzione ${ }^{1}$}

Le emergenze migratorie ${ }^{2}$ tendono a essere viste come il risultato di un'anomalia strutturale, di tipo sociale, politico o economico (Malkki, 1992, p. 33). Si tratta, tuttavia, di una visione recente. Tra la fine della Prima Guerra Mondiale e immediatamente dopo la Seconda Guerra, I'idea di rifugio non faceva riferimento a un'anomalia sociale o economica che le dava origine, ma alla situazione del rifugiato nel suo essere sradicato, uprooted, e perciò una minaccia sociale, politica e, soprattutto, morale (Simpson, 1939; Cirtautas, 1957) ${ }^{3}$. Questa visione del rifugiato come patologia dello sradicato (Malkki, 1992, p. 33) è scomparsa nei recenti studi, senza però scomparire l'idea del rifugiato come un problema.

La prospettiva del rifugiato come una emergenza o una catastrofe costituisce ancora l'elemento costitutivo di molti studi sulle migrazioni forzate. Non è più un'aberrazione morale e politica, ma un problema sociale, economico e politico (a volte psicologico o di salute pubblica) che deve essere risolto. Contrariamente a questa visione, le migrazioni possono essere interpretate anche come una forma creativa alla quale gli individui ricorrono per affrontare situazioni avverse o adattarsi a repentini cambiamenti nel loro contesto. La prima prospettiva insiste sugli scenari di sofferenza o vulnerabilità che i migranti affrontano. In questa logica categorie come "migrazione forzata" acquistano rilevanza. Il presupposto di questa visione è concepire il legame a un luogo, una terra o una nazione come condizione d'identità o dignità di una persona. Lo spostamento geografico, inoltre, è interpretato come una forma di anomalia che rende gli individui vulnerabili e non come una forma mediante la quale

1 Traduzione in italiano di un articolo originalmente pubblicato con il titolo "Globalização, mobilidade humana e criatividade: desafiando categorias a partir de três casos de migarção forçada in Angola", nel libro: VASCONCELOS, Ana Maria Nogales; BOTEGA, Tuíla (orgs.). Política migratória e o paradoxo da globalização. Porto Alegre: EDIPUCS; Brasília: CSEM, 2015, p. 169-188. Traduzione di Roberto Marinucci.

2 Questo articolo si concentra sulle cosiddette migrazioni forzate, una sottocategoria del fenomeno migratorio che fa riferimento a quel tipo di mobilità in cui gli attori sono costretti, direttamente o indirettamente, ad abbandonare i loro luoghi abituali di residenza: rifugiati, sfollati (deslocados), esiliati, rimpatriati e retornados (coloro che ritornano volontariamente). Queste categorie non saranno discusse singolarmente nel corso del testo, ma sono considerate nel loro insieme come diverse modalità delle migrazioni forzate. A volte useremo i termini mobilità e migrazioni come sinonimi, anche se consideriamo la mobilità come un fenomeno più globale e generico di movimento di persone, e la migrazione come una forma istituzionale di riferirsi specificamente a un certo tipo di mobilità. In ogni caso, il testo si concentra sulle migrazioni forzate, anche quando non sono esplicite. Per una discussione o concettualizzazione sul movimento di persone si veda, ad esempio, Frello (2008). E per una critica del concetto di migrazione o mobilità si veda, ad esempio, Glick-Schiller, Salazar (2013).

3 John Simpson ha anche scritto in un rapporto che "politicamente sradicato [il rifugiato] può immergersi nella malavita, nel terrorismo e nel crimine; e in ogni caso è sospettato d'irresponsabilità politica e mette in pericolo la sicurezza nazionale" (Simpson, 1939, p. 9, traduzione nostra). 
individui o gruppi umani ribadiscono la loro dignità e affrontano situazioni politiche ed economiche avverse.

Le agenzie internazionali e le organizzazioni non governative - e anche alcuni governi - hanno bisogno di utilizzare classificazioni per gestire l'arrivo improvviso di moltitudini di persone provocato da episodi di violenza o disastri naturali. Questa necessità meramente burocratica e amministrativa tende a sedimentarsi e con il passar del tempo acquisisce lo status di metodo di produzione di categorie e tipologie sulla mobilità umana: rifugiati, sfollati (deslocados), rimpatriati o retornados. Altre sottocategorie vengono create quando quelle citate non descrivono sufficientemente bene la realtà quotidiana dei migranti: migrazione forzata o involontaria e migrazione volontaria. Le ricerche e gli studi sulla mobilità umana non guardano sempre in modo critico a queste classificazioni. Tali categorie, progettate esclusivamente a scopi pratici, cioè, per gestire episodi di violenza o disastri naturali che causano grandi movimenti di persone, spesso non hanno una base empirica solida, o sono semplicemente adattate e assunte acriticamente nel contesto della ricerca accademica. II risultato è che si entra in un circolo vizioso: la necessità di gestire la mobilità umana porta alla creazione di tipologie e classificazioni che sono riprese dalla ricerca accademica e ricevono lo status normativo di descrittori della realtà, che, a loro volta, vengono riassorbiti dai policy-makers.

Un'alternativa sarebbe quella di adottare la prospettiva che vede le migrazioni come una risorsa che un individuo o un gruppo di persone ha a disposizione per affrontare circostanze sfavorevoli, presumendo, anche nel peggiore degli scenari, un margine decisionale. In questo senso, le categorie come "migrazione forzata" possono essere criticamente ripensate. E' proprio su questo tema che il presente articolo intende riflettere: il processo di produzione di categorie, sia da parte dei policy-makers, sia in alcuni studi sulla mobilità umana, sottolineandone alcune difficoltà e limitazioni.

Il punto di partenza è la percezione che l'eccessiva fissazione su categorie come "rifugiati" o "retornados" non solo rende invisibili altre forme di "retorno" o di migrazione motivata dalla ricerca di migliori "condizioni di vita", che persistono al di là delle etichette ufficiali, ma aumenta altresì sugli stessi migranti quella tensione che già presuppone il fronteggiare gli ostacoli alla mobilità (Lubkemann, 2008). Così, partendo dal concetto di migrazione come un modo creativo al quale individui o gruppi ricorrono per affrontare circostanze avverse, sosteniamo che nell'etichettare un individuo come rifugiato o rimpatriato si corre sempre il rischio d'interrompere processi di trasformazione / adattamento più profondi in cui la migrazione è solo un'espressione o una fase del ciclo completo. Questa prospettiva, come vedremo in seguito, ha ripercussioni sulla ricerca scientifica, ma anche sulla progettazione delle politiche d'intervento. 
Questo articolo raccoglie, brevemente, alcune riflessioni sull'esperienza di ricerca in Angola negli ultimi sette anni, nell'ambito della mobilità umana. Non presenteremo qui i risultati di queste ricerche, ma alcune riflessioni basate su esempi concreti. I tre esempi sono: angolani retornados dallo Zambia; angolani rimpatriati dalla Repubblica Democratica del Congo; e sfollati interni nella provincia centrale di Huambo, in Angola. L'inizio delle ricerche risale al $2007^{4}$.

\section{Categorie, classificazioni e soggettività}

In un conosciuto dibattito avvenuto nel Journal of Refugee Studies nel 2007, molti ricercatori sociali si chiedevano quale fosse l'oggetto di studio delle cosiddette migrazioni forzate. Le opinioni potevano essere suddivise in questo modo: quelli che focalizzavano $i$ rifugiati nel modo in cui sono definiti dalle Nazioni Unite e dalla Convenzione di Ginevra (Hathaway, 2007); coloro che mettevano in evidenza l'importanza d'includere anche le vittime di violenza che abbandonano le proprie terre, ma senza attraversare necessariamente la frontiera, ossia, gli sfollati, noti anche come IDPs ${ }^{5}$ (Cohen, 2007); infine, quelli che pretendevano annoverare non solo le conseguenze, ovvero i migranti forzati, ma anche i contesti sociali e politici che causano questi movimenti (DeWind, 2007).

Bakewell (2008), in un articolo pubblicato un anno dopo nella stessa rivista, riprendeva il dibattito criticando le posizioni precedenti. Tutte avevano qualcosa in comune: si concentravano su alcune categorie di persone, siano esse sfollate o rifugiate, che erano sotto la protezione di agenzie internazionali e che, di conseguenza, erano oggetto di un trattamento specifico (Bakewell, 2008, p. 440). E' la condizione specifica di queste persone, i loro diritti, i loro bisogni - fisici, sociali, giuridici - che costituirebbe l'oggetto della ricerca. Ciononostante, la definizione dei bisogni dei rifugiati, il loro status giuridico e umanitario, ha origine da un intervento amministrativo.

Le ricerche, con una certa frequenza, assumono acriticamente questi dati e categorie prodotti in contesti amministrativi, anche per l'inevitabile relazione con la Convenzione relativa allo status dei rifugiati, firmata a Ginevra nel 1951 (Convenzione del 1951) ${ }^{6}$, che ha inaugurato un regime giuridico per la protezione dei rifugiati, stipulando i loro diritti e doveri e salvaguardando la sovranità degli Stati in merito al rispetto delle leggi del paese di destinazione.

\footnotetext{
${ }^{4}$ I dati sono stati raccolti nell'ambito di diversi progetti di ricerca e contatti con Agenzie umanitarie, che saranno citati nel corso del testo.

5 Sigla in inglese per Internally Displaced People (sfollati interni).

6 In effetti la Convenzione riunisce una documentazione precedente sulla protezione dei rifugiati, come quella del 12 maggio 1926, del 30 giugno 1928, o delle Convenzioni del 28 ottobre 1933 e del 10 febbraio 1938, e del Protocollo del 14 settembre 1939, e della Costituzione dell'Organizzazione internazionale dei rifugiati.
} 
Un elemento chiave della Convenzione è l'Alto Commissariato delle Nazioni Unite per i Rifugiati (UNHCR), che

è incaricato di vigilare all'applicazione delle convenzioni internazionali intese a garantire la protezione dei rifugiati e che il coordinamento effettivo delle misure prese per risolvere tale problema dipende dalla cooperazione degli Stati con I'Alto Commissariato. (Preambolo della Convenzione 1951)

Come risultato dei successivi flussi migratori, in particolare quelli forzati, e della necessità di una migliore gestione e contenimento dei drammi umani si installò lentamente il sistema di campi profughi, che, nel corso del tempo, è diventato, di fatto, un regime. Malkki (1985) definisce i campi profughi come una "tecnologia di cura e controllo" (Malkki, 1985, p. 51), vale a dire, una tecnologia di potere che mira a gestire lo spazio e la circolazione di persone che si trovano "fuori ordine" (Malkki, 1992, p. 34). Per i suoi abitanti è una struttura di potere con un regime giuridico e amministrativo definito, un territorio artificiale e poroso, e un sistema di controllo sociale. ONG, agenzie internazionali e fondazioni gestiscono i campi, direttamente o indirettamente. Convenzioni, normative, direttori e la cultura di queste organizzazioni sono i riferimenti da cui sono elaborati i diritti e i doveri di coloro che risiedono nei campi, ma anche le pratiche con le quali essi devono relazionarsi, collaborare, confrontarsi e, alle volte, ribellarsi. E', infatti, un regime (Inhetveen, 2010). Oltre a funzionare come un sistema educativo ad hoc, più convenzionale, fornisce anche una formazione nell'ambito dei diritti umani, genere e educazione alla pace.

Un esempio è il campo di Maheba, a ovest della Repubblica dello Zambia, che ha accolto rifugiati angolani per decenni ${ }^{7}$. Con la fine della guerra civile, nel 2002, e come risultato di un accordo tripartito tra le Nazioni Unite, attraverso l'Alto Commissariato delle Nazioni Unite per i Rifugiati, il governo della Repubblica di Angola e il governo della Repubblica dello Zambia, è stato avviato il processo di smantellamento del campo attraverso un programma di rimpatrio.

Le organizzazioni usavano non solo le tecnologie d'intervento in base ai beneficiari e alla loro cultura istituzionale, ma anche in base alla capacità finanziaria e all'esperienza gestionale. Un processo di classificazione è stato sviluppato. Quelle organizzazioni che lavorano sia in Angola che in Zambia dovevano classificare / categorizzare i loro oggetti d'intervento in base alle loro pratiche di gestione amministrativa. Alcuni individui erano amministrativamente definiti in base alle circostanze in cui si trovavano. Ad

\footnotetext{
Solamente in Zambia si stima che il numero sia stato pari a 211 mila, distribuiti in diversi campi. Il campo di Maheba era uno dei più grandi. Cfr.: United Nations High Commissioner for Refugees (UNHCR), Angola signs repatriation agreements with UNHCR, Namibia and Zambia, Geneva, 28 November 2002. Per una visione completa dei campi profughi in Zambia che accoglievano rifugiati, si veda, ad esempio, Inhetveen (2010).
} 
esempio, coloro che fuggivano dalla guerra e attraversavano il confine erano rifugiati; coloro che fuggivano dalla guerra ma senza attraversare il confine e si stabilivano in campi o centri di trattenimento nel proprio paese erano gli sfollati; quelli che tornavano volontariamente, dopo la guerra, alle terre di origine erano retornados; quelli, invece, che tornavano in modo forzato erano rimpatriati. C'era anche la situazione di quegli angolani, parenti di politici altolocati e dirigenti delle forze armate, che sono fuggiti in Zambia durante la guerra. Non furono annoverati nella categoria di rifugiati, dato che non attraversarono la frontiera a piedi o con I'aiuto delle Nazioni Unite, ma attraverso mezzi convenzionali come, ad esempio, gli aerei. Si stabilirono in case private e, non appena passò la sensazione di pericolo, tornarono immediatamente. Questi appartenevano alla categoria degli espatriati (expatriados), usata per definire i cosiddetti migranti ad alto reddito, o migranti di lusso (Gatti, 2009; Fechter, 2007).

Le categorie "politico" e "amministrativo" sono state il punto di partenza di molti studi accademici che hanno dato ad esse lo status epistemologico, ma anche aperto nuovi campi di studio: le cause della mobilità dei rifugiati, il problema di adattamento e reinserimento dei retornados (Kaun, 2008), il problema dei giovani (Auerbach, 2010), delle donne, dei bambini e degli ex soldati (Hansen, 1999). II rifugiato, da categoria politica e amministrativa, si trasforma in un'entità sociale con un percorso e con attributi propri, e con una storia, di solito violenta; è, inoltre, isolato da quel contesto sociale e politico che rende intellegibile parte della sua condizione di rifugiato. Questa prospettiva si basa su scelte teoriche e metodologiche che non solo corrono il rischio di fuorviare la realtà vissuta dagli stessi rifugiati, ma anche, in casi estremi, di contenerli in una campana di vetro, diventando, così, un'ulteriore avversità da affrontare.

I governi dei paesi che ricevono rifugiati, o nei quali essi devono tornare, spesso usano le stesse categorie o terminologie del campo. Da un lato, gli sfollati sono per lo più ammassati in campi lontani dalle zone residenziali per non disturbare il funzionamento degli encontrados. Si insiste, altresì, sull'uso delle categorie rimpatriati o retornados, che, in molti casi, non solo stigmatizzano, ma permettono ai funzionari delle pubbliche amministrazioni di utilizzare le stesse categorie per creare ostacoli burocratici. Un esempio concreto potrebbe essere la difficoltà di ottenere i documenti nazionali o la carta d'identità per i rimpatriati. Un altro, il riconoscimento ufficiale dei diplomi accademici o professionali dei retornados, che sono stati acquisiti mentre erano all'estero ${ }^{8}$.

\footnotetext{
Interviste con angolani retornados dallo Zambia hanno mostrato in particolare la difficoltà di riconoscimento dei diplomi d'istruzione ottenuti nei campi profughi o nel sistema educativo del paese dove risiedevano come rifugiati (interviste a angolani retornados dallo Zambia e dalla Repubblica Democratica del Congo).
} 


\section{Mobilità, creatività e vita quotidiana}

Quanto detto sopra mette in evidenza le opzioni teoriche sul tema delle migrazioni. Como abbiamo già detto, le migrazioni possono essere viste come un modo di affrontare situazioni avverse e, da questo punto di vista, vi sono sempre margini per la decisione di non emigrare. Sia Jónsson (2011) che Lubkemann (2008) e Adey (2006) discutono sui margini della non-mobilità e sulle implicazioni teoriche della percezione della mobilità. La mobilità, anche nei casi di guerra, può essere vista come una risorsa a che individui o gruppi ricorrono per affrontare le criticità contestuali (Lubkemann, 2008; Bakewell, 2008). La prima conseguenza di questa prospettiva è mettere tra parentesi la divisione tra migrazione "forzata" e migrazione "involontaria".

Un esempio dell'uso dei limiti di queste categorie sono i flussi migratori tra le due parti del confine Angola-Zambia già menzionati anteriormente, specialmente nella regione dell'Alto Zambesi. Fino all'introduzione della frontiera politica alla fine del XIX secolo dai portoghesi e dagli inglesi era un subregione in cui la mobilità delle persone, di tradizione secolare, avveniva da una parte all'altra, in particolare per motivi commerciali (Vansina, 1966; Von Oppen, 1994). Le alleanze dei leader politici si estendevano su entrambi i lati del confine. Anche dopo l'introduzione dei confini politici, i movimenti non si interruppero. Quando all'inizio del XX secolo fu costruita la ferrovia Benguela, che attraversa I'Angola dalla costa atlantica al confine orientale, molti zambiani videro un'opportunità di lavoro e iniziò un flusso migratorio verso l'Angola.

Quando, sempre nel corso del XX secolo, le autorità coloniali imponevano sulle popolazioni angolane un eccesso di tributi - anche sulle case modeste, come nel noto caso dell' impostos da cubata (Neto, 1997, p. 435) -, molti decisero di emigrare e rifugiarsi nello Zambia, dove la pressione fiscale era meno pesante. Anche quando iniziò la guerra anticoloniale contro i portoghesi, molti angolani cercarono rifugio nello Zambia, sia per fuggire dalla guerra civile, sia come punto d'appoggio militare per combattere le truppe portoghesi. Durante la guerra civile angolana tra il MPLA (Movimento Popolare di Liberazione dell'Angola), di ispirazione marxista, che governa l'Angola dal 1975, e I'UNITA (Unione per l'Indipendenza Totale dell'Angola), inizialmente maoista, ma che in seguito si alleò con le potenze occidentali, la popolazione e i militari si rifugiarono in Zambia. Alla fine della guerra civile, molti profughi, specialmente quelli che vivevano nei campi, tornarono in Angola.

In questo costante andirivieni, le popolazioni frontaliere vedevano nella possibilità di emigrare una risorsa alla quale ricorrere se le strategie di gestione delle situazioni avverse superassero la loro capacità di resistenza. Tuttavia, non tutti gli abitanti dei villaggi di confine attraversavano la frontiera. Un esempio concreto è quello della regina Nhakatolo, la leader dei Luvales, un gruppo 
etno-linguistico dominante nella provincia di Moxico, regione orientale dell'Angola, che si rifugiò a Luanda, a centinaia di chilometri di distanza, a differenza dei leader di altri gruppi, come i Lunda o Bundo, che attraversarono il confine.

Pertanto, quando si parla di migrazione forzata, e in particolare delle sue cause, è importante prendere in considerazione altre categorie, come la classe sociale, il potere politico e l'appartenenza, e altre dimensioni, come quella economica e la percezione territoriale. Concentrandosi oltremisura sulla migrazione forzata, non solo si cancella la capacità di agency degli attori sociali, ma si eclissano anche alcune dimensioni, probabilmente importanti, del fenomeno migratorio che questa categoria non racchiude. Una ipotesi potrebbe essere quella di interpretare questi spostamenti come una sfida alla concezione eccessivamente rigida e classica della frontiera, in parte come risultato di lotte europee del XVII e XVIII secolo, che hanno portato alla definizione di confini, successivamente trasferiti in Africa. Quindi, invece di sostenere che sono le frontiere che definiscono politicamente una persona, è possibile affermare che le dinamiche sociali mettono in discussione, attraverso frequenti e successivi passaggi, una sorta di definizione politica del territorio (Giles-Vernick, 1999).

Un altro esempio dei limiti delle categorie deriva dal contatto con i rimpatriati angolani, questa volta nel nord dell'Angola, al confine con la Repubblica Democratica del Congo (RDC) ${ }^{9}$. L'azione delle forze di polizia angolane ha portato a una tragedia umana: migliaia di congolesi che lavoravano come garimpeiros in aree minerarie dell'Angola orientale sono stati espulsi senza che il governo osservasse le procedure legali e le convenzioni internazionali di rimpatrio. Per rappresaglia, il governo della RDC ha rimpatriato forzatamente migliaia di angolani che risiedevano nel paese confinante, in alcuni casi da decenni. Di conseguenza, migliaia di angolani, oltre 40.000 secondo alcune agenzie umanitarie ${ }^{10}$, sono improvvisamente arrivati in quelle zone di confine. Lo status di rimpatriati assegnato a questi soggetti da agenzie umanitarie è stato assunto dal governo, in particolare dalle amministrazioni locali, e utilizzato come misura per definire i "nuovi soggetti". Mentre i servizi di migrazione si affannavano in dispute burocratiche per definire chi fosse angolano e chi un possibile infiltrato congolese, l'integrazione della maggior parte di questi rimpatriati, a livello sociale, avveniva con un certo successo. Alcuni fattori hanno contribuito al buon esito dell'integrazione: il flusso di migranti tra i due paesi non si era mai interrotto; i due lati del confine settentrionale parlavano la

9 Il contatto con questi retornados è avvenuto nel contesto di attività di valutazione di progetti finanziati da Weltkirche (Germania) e Caritas International e realizzati dalla Caritas di Angola. I progetti miravano ad assistere i retornados in vari modi (cfr. Inglês, 2012).

10 Questo numero è stato fornito dalla Caritas Angola (rapporto 2010) e dal Servizio dei Gesuiti per i Rifugiati (JRS) - Angola. Informazioni JRS /2011. 
stessa lingua - il Lingala - e appartenevano allo stesso gruppo etno-linguistico il Bakongos; il flusso di prodotti e gli scambi commerciali su entrambi i lati del confine; I'uso dell'idioma Lingala come lingua veicolare quotidiana - molto più del portoghese, la lingua dominante in Angola e poco utilizzata nella regione -; il consumo di prodotti culturali, in particolare la musica e i programmi radiofonici e televisivi. Nonostante questo contesto che facilitò l'integrazione, i rimpatriati hanno affrontato diversi problemi burocratici. A causa dell'uscita forzata dalla RDC, non ebbero il tempo sufficiente per provvedere alla documentazione che comprovasse la cittadinanza angolana o la residenza nella RDC. Così, I'Alto Commissariato delle Nazioni Unite per i Rifugiati (UNHCR), entità che interviene per risolvere la situazione giuridica dei rimpatriati, fu colto alla sprovvista. Senza il tempo sufficiente per preparare il processo di rimpatrio, i rimpatriati si sono ritrovati nella situazione di indocumentados (Khosravi, 2007), senza essere in grado di dimostrare che erano angolani e, in alcuni casi, che erano stati rifugiati in Congo.

Anche in questo caso, il problema sembra superare le questioni specifiche della mobilità, come le cause o i problemi d'integrazione dei retornados, e si delineano questioni più globali, come ad esempio: l'interazione tra identità locale e trans-locale - come vincolo sociale di individui o gruppi che si concretizza nella condivisione di determinati legami sociali, indipendentemente dalle frontiere politiche che attraversano queste comunità (Haddad, 2003); la cittadinanza, intesa come l'essere soggetto di diritti e doveri in relazione a una comunità politica all'interno di uno Stato-nazione (Habermas, 1995; Soysal, 2000); e l'esercizio del potere attraverso un governo stabilito (Dillon, 1995, p. 324). E' nell'intersezione di questi elementi - cultura, politica e potere - che si può pensare la mobilità umana. A volte può significare la riconfigurazione delle identità in determinati contesti, siano essi locali, trans-locali o nazionali. In altri casi possono essere collegati alla ridefinizione della cittadinanza e al contesto politico in cui si muovono individui e gruppi. In altri ancora, è l'esercizio del potere che si contrae e, in questo caso, vi è l'uso della violenza, compresa quella fisica, come modo di esercitare la disciplina e il controllo sociale e stabilire una gerarchia tra individuo e comunità. Inoltre, quando questo stesso potere si assottiglia, vengono allora utilizzate pratiche giuridiche o sistemi legali per continuare a esercitare quel controllo sociale. La migrazione in questo contesto può anche essere una "migrazione reattiva", come la definisce Anthony Richmond (1993), cioè una forma attiva di azione sociale.

Quest'ultimo aspetto rimanda la questione della migrazione al contesto dell'interazione sociale, a come individui e gruppi umani interagiscono per rendere il loro mondo sociale "possibile", nonostante i condizionamenti. La migrazione, come già sottolineato, anziché essere interpretata solo come una conseguenza o unicamente per quegli aspetti drammatici vincolati alla mobilità, 
può essere vista come una risorsa flessibile per rendere possibile la quotidianità. La nozione di homo migrans di Bade (1994) mette in evidenza la capacità umana di utilizzare la migrazione come un modo di ricreare la vita quotidiana, benché in un altro luogo. Così, nozioni come adattamento e creatività (Joas, 1996) aiutano a comprendere meglio il fenomeno sociale della migrazione piuttosto che la fissazione su categorie come retornado, rimpatriato o sfollato.

Altri due esempi possono illustrare ciò che abbiamo appena detto. II primo si riferisce all'esperienza di sfollati agli inizi della guerra civile a Huambo ${ }^{11}$, in Angola; I'altro ai retornados angolani che si sono rifugiati in Zambia.

Il primo caso riguarda gli insediamenti per sfollati installati nei dintorni della città di Huambo, che, dopo la ripresa della guerra civile nel 1998, ha accolto persone in fuga da villaggi e paesini della provincia centrale di Huambo. Qui facciamo riferimento solo all'insediamento del Kassequel, noto come campo per gli sfollati' ${ }^{12}$. Le descrizioni dell'arrivo degli sfollati nei campi sono drammatiche: la fuga inizia all'alba, con bambini e anziani; percorrono più di 100 chilometri, senza nulla da mangiare e da bere; nella città di Huambo, dove arrivano stanchi e affamati, sono ricevuti da organizzazioni non governative e agenzie internazionali per il primo soccorso e, successivamente, si stabiliscono in campi di trattenimento temporaneo, campi di transizione, e in tende da campo. Le condizioni dei campi di transizione durante i primi mesi erano insalubri. La diffusione di malattie infettive era una minaccia costante.

La violenza dei combattimenti non facilitava la distribuzione degli aiuti umanitari. Dopo alcune settimane, le agenzie internazionali e le ONG, in collaborazione con il governo locale, hanno deciso di trasferire il campo di trattenimento temporaneo negli insediamenti, uno dei quali fu proprio quello di Kassequel. Con il passar del tempo gli sfollati si stavano adattando alle nuove condizioni di vita, innanzitutto mediante la costruzione di case, ancora con materiali precari, ma più resistenti alla pioggia e ai venti rispetto alle tende da campo; alcuni uomini lavoravano nella costruzione di abitazioni, mentre alcune donne facevano commerci di strada o subaffittavano terreni per coltivare piccoli orti. Alcuni capi villaggio, che si erano trasferiti, hanno mantenuto la

${ }^{11}$ La guerra civile angolana, che oppose il governo guidato dal MPLA e I'UNITA, si è conclusa nel 2002. Nel 1991, è stato firmato un accordo di pace (Accordo Bicesse), che prevedeva la fine del conflitto militare e l'introduzione del sistema multipartitico. Le prime elezioni, tenutesi nel 1992, furono vinte dall'MPLA. L'UNITA non ha riconosciuto i risultati elettorali, il che ha provocato la ripresa del conflitto. Nonostante i tentativi di porre fine alla guerra ed alcune brevi interruzioni, nel 1998 i combattimenti si sono intensificati ed hanno prodotto migliaia di sfollati e rifugiati. II conflitto si è concluso quando il leader dell'UNITA, Jonas Savimbi, è stato ucciso. Gli accordi di pace sono stati ripresi e le due parti hanno firmato il Memorandum de entendimento do Lwena, ponendo così fine a quasi 27 anni di guerra civile

12 La differenza tra insediamento e campi profughi o rifugiati è che nel primo caso si tratta di uno spazio già esistente, già abitato, che viene ampliato per ospitare gli sfollati. Al contrario, i campi profughi sono spazi appositamente creati, di solito in aree aperte o terreni non edificati. 
loro posizione sociale e, quindi, fungevano da intermediari nel gruppo, con una autorità riconosciuta dalle ONG e dagli attivisti locali. Tali leader hanno promosso la costruzione di fontane e scuole per bambini, erette dai genitori con I'assistenza tecnica e materiale delle ONG e delle agenzie umanitarie. II governo ha fornito corsi di formazione per insegnanti e materiale scolastico.

Quando è finita la guerra, è sorto il problema dello smantellamento degli insediamenti. La politica del governo era favorevole al regresso degli sfollati nei loro villaggi di origine. Anche alcune agenzie umanitarie erano di questo parere, sostenendo che nelle aree rurali potevano lavorare nei campi e avere cibo sufficiente per soddisfare il fabbisogno (Lari, 2004). Tuttavia, mentre si discuteva sul tema, alcune persone avevano già deciso di ritornare, ma con differenti modalità. Alcuni avevano scelto di ritornare in modo definitivo; altri, al contrario, trascorrevano solo alcuni periodi di tempo nei villaggi, soprattutto durante il periodo della lavorazione, della semina e della raccolta, passando il resto del tempo a Huambo. Ci sono stati casi in cui le famiglie hanno adottato altre strategie per affrontare la nuova situazione, come, ad esempio, nel caso delle famiglie che avevano deciso di lasciare a Huambo i figli più giovani con i bambini in età scolare, mentre i genitori e i figli più piccoli erano tornati al villaggio.

Le diverse modalità di retorno indicano un adattamento al contesto, ma anche una trasformazione sociale. Così, la nozione di campo e città è stata fusa attraverso l'uso degli spazi con funzioni differenti. Anche la nozione di "terra d'origine", categoria politica e amministrativa da cui deriva il termine retorno o rimpatriato, è stata ripensata: la nozione di costruzione sociale ha acquisito rilevanza a discapito dell'essenzializzazione del concetto. Così, la terra di origine cessa di essere un luogo e diventa uno spazio, nel senso indicato da Michel Certeau (1980), secondo il quale lo spazio

è l'effetto prodotto dalle operazioni che lo guidano, lo circoscrivono e lo circostanziano, lo temporalizzano e lo fanno funzionare come unità polivalente di programmi conflittuali o di prossimità contrattuali... A differenza del luogo, lo spazio non ha dunque né l'univocità né la stabilità di qualcosa di circoscritto. (Certeau, 1980, p. 208)

Può anche essere una strategia per affrontare possibili focolai di violenza che possono sorgere o semplicemente per mantenere le due aree di riferimento, la città e la campagna, come possibili risorse per gestire eventuali imprevisti. Ad esempio, con la crisi petrolifera, che è la base dell'economia angolana, la migrazione verso aree rurali può essere una risorsa per far fronte alla riorganizzazione della produzione nelle città. Altre volte, la siccità o altre calamità naturali possono indurre il movimento inverso. Laddove in termini 
burocratici questa eventualità può essere vista come un'anomalia, nella vita quotidiana delle persone rappresenta una normalità.

L'altro esempio viene dall'esperienza dei retornados dallo Zambia in Angola $^{13}$. Alcuni si sono diretti alla città di Kwito, capitale della provincia angolana di Bié, nell'Angola centrale. Questi retornados sono per lo più ex soldati dell'UNITA e civili che prima della guerra erano sostenitori dell'UNITA, i quali, nel corso della fuga, sono fuggiti verso zone controllate dal partito per timore di rappresaglie da parte delle truppe o dei simpatizzanti del governo. Alla fine del conflitto, nel 2002, e in seguito a un accordo tripartito tra i governi di Angola e Zambia e l'Alto Commissariato delle Nazioni Unite per i Rifugiati, è iniziato un processo di retorno, tecnicamente noto come "rimpatrio volontario". E' stato in questo modo che alcuni retornados, con l'aiuto logistico dell'Alto Commissariato e del governo angolano, sono arrivati a Kwito. L'ostilità di alcuni abitanti di Bié verso i retornados - perché erano nemici antichi - era ancora visibile, nonostante gli accordi di pace. I retornados hanno chiesto al governo della città di concedere loro un terreno nelle vicinanze di Kwito dove poter erigere un quartiere. Nel terreno ottenuto hanno costruito il quartiere denominato, ironicamente, Bairro da Zâmbia. Dal 2003, i retornados che arrivavano dallo Zambia e che non riuscivano a trovare un posto dove vivere a Bié, rimanevano nella città di Kwito, nel Bairro da Zâmbia.

Le interviste attestano la presenza di un servizio di accoglienza per i nuovi arrivati e alcune ostilità da parte di antichi residenti di Kwito. I segni della guerra erano ancora presenti. Kwito è stata una delle città in cui sono stati combattuti diversi scontri militari, rimanendo assediata alcuni mesi dai soldati dell'UNITA. Un sentimento di ostilità nei confronti dei retornados era ancora presente in alcuni degli abitanti di Bié, secondo le interviste. Per i retornados, il Bairro da Zâmbia è diventato non solo uno spazio di resistenza contro I'ostilità di altri angolani, ma anche uno spazio di ricostruzione della memoria - della guerra e della lotta politica. Così, mentre gli studi sulle migrazioni potevano insistere su questioni come la reintegrazione dei retornados, nel quotidiano di questi si trattava di ricostruire la memoria per adattarla alle pratiche quotidiane e per rendere possibile la ricostruzione di una routine.

${ }^{13}$ I dati riferiti nel presente paragrafo sono stati raccolti nell'ambito del progetto "Refugee Repatriation and Local Politics in Angola Conflict and Creativity Following the Return of Chiefs and Party Functionaries", diretto dal Professor Katharina Inhetveen (il progetto è stato svolto fino al 2012 nell'Università Ludwig-Maximilians di Monaco di Baviera e in seguito presso l'Università Bundeswehr di Monaco) e realizzato tra il 2010 e il 2014. La ricerca sul campo in Zambia e Angola si è svolta nel 2010 e 2012. Il progetto fa parte del programma Priority Programme 1448 da Deutsche Forschungsgemeinschaft (DFG); è un programma allargato di ricerca dal titolo "Adaptation and Creativity in Africa -Technologies and Significations in the Production of Order and Disorder". 
Alcuni elementi raccolti nel lavoro sul campo in Kwito sollevano domande sull'utilizzo della categoria retornado. Per i retornados nel Bairro da Zâmbia, l'esperienza di vita nel campo profughi non è stata solo una esperienza di sofferenza e vulnerabilità, ma anche di resistenza contro il potere politico. Dalle interviste si deduce che hanno ricostruito per sé una narrazione in cui l'esperienza del campo profughi era parte di una protesta contro la centralizzazione del potere politico. Quindi, il soggiorno all'estero è stato una specie di esilio, simile a quello raccontato da Liisa Malkki in relazione ai rifugiati hutu in Tanzania (Malkki, 1995). Per loro, essere stati rifugiati non significa solo una fuga dalla morte imminente a causa della violenza della guerra civile; non è stata una migrazione forzata o involontaria; è stata, al contrario, una maniera di contestare il potere politico. Allo stesso modo, i retornados hanno interpretato il rimpatrio o il retorno come la fine della resistenza. Il significato di questo discorso, nonostante le implicazioni ideologiche, era soprattutto pratico in quanto derivava dalla necessità della ricostruzione della vita quotidiana in cui le esperienze passate sono integrate o incorporate in un discorso coerente e plausibile. In questo modo non solo hanno dato un senso alle loro vite e al loro universo sociale, ma hanno anche integrato le loro esperienze nella stessa storia dell'Angola.

Una delle implicazioni di questa prospettiva è guardare al rifugiato o al retornado non solo come un soggetto bisognoso di protezione (fisica e giuridica) delle istituzioni internazionali, isolandolo come categoria, ma situarlo in un processo più ampio e più complesso di riconfigurazioni sociali e politiche. Implica anche un ripensamento del concetto stesso di mobilità ${ }^{14}$ : è possibile isolare tale fenomeno dagli altri fenomeni a esso adiacenti, come i conflitti, le riconfigurazioni sociali, politiche ed economiche? Significa anche mettere nuovamente in discussione aspetti che contengono residui delle teorie economiche classiche e della New Economy che insistono sulle cause e le motivazioni della mobilità. Infine, questa prospettiva può apportare implicazioni metodologiche ed epistemologiche: nel primo caso, si passa da una visione esterna e quantitativa del fenomeno della migrazione a una dimensione longitudinale e qualitativa; sarà necessario considerare le interpretazioni che lo stesso rifugiato o retornado fa della sua situazione e dei suoi spostamenti. Nel secondo caso, si tratta d'investigare ulteriormente il tipo di conoscenza che gli studi migratori producono: fino a che punto le categorie prodotte o riprodotte da questi studi tentano di comprendere e spiegare il fenomeno della mobilità oppure, andando oltre, producono questi stessi fenomeni? In altre parole, fino a che punto le categorie prodotte per spiegare questi fenomeni non li offuscano ulteriormente, sottraendoli dai contesti che li rendono comprensibili?

\footnotetext{
${ }^{14}$ Per una discussione critica del concetto di mobilità, si veda, ad esempio, Holert, Terkessidis (2005).
} 


\section{Globalizzazione, riconfigurazioni e limiti della scienza}

Nonostante i dubbi sull'utilità delle categorie rifugiato e retornado, in termini pratici il ricorso ad esse è inevitabile, soprattutto quando si tratta di gestire le crisi umanitarie, sia da parte dei governi che delle agenzie internazionali. Quando si ha a che fare con migliaia di persone in movimento, è quasi inevitabile ricorrere a classificazioni o a categorie per ridurre la complessità, fornire assistenza legale e sostegno umanitario. Tuttavia, si può sostenere che le classificazioni possono essere utili nella gestione, ma problematiche nel contesto della ricerca. Molti ricercatori sociali sono anche consulenti di agenzie umanitarie e governi, e il confine tra le due attività quella di ricerca e di consulenza -, sono tenui; quindi, le categorie prodotte nell'ambito della gestione e dell'amministrazione sono trasferite nell'ambito scientifico e viceversa ${ }^{15}$. In aggiunta, molte ricerche sono finanziate da agenzie che finanziano anche programmi di aiuti umanitari, e le agenzie umanitarie si rivolgono spesso a ricercatori sociali per l'elaborazione di progetti, studi e valutazioni di programmi. Sembra che il problema sia un certo "rifugiatocentrismo" o "migrante-centrismo" (Landau, 2007, p. 347). Il fatto di focalizzare in modo esagerato la figura del rifugiato come categoria, specialmente nel contesto delle migrazioni forzate, sradica gli individui dai contesti sociali e storici in cui i movimenti hanno luogo. Sorge, pertanto, la necessità di studi sui rifugiati che includano altre discipline e, quindi, incorporino altre categorie per analizzare e spiegare il fenomeno migratorio.

D'altro canto, il contesto sociale e politico che genera migrazioni forzate, come sottolinea Dewind (2007), è vasto e molto complesso. Sarebbe necessario includere tutte le scienze sociali per percepire, per esempio, ciò che ha portato gli angolani a rifugiarsi in Zambia e nel Congo, oppure i mozambicani in Sud Africa, o i ruandesi in Kenya, o più recentemente i siriani in Germania. Una possibile pista per uscire da questo vicolo quasi cieco è guardare alle migrazioni come a un fenomeno umano che può essere un semplice segno dello "stare del mondo". Pertanto, invece di presupporre che le migrazioni siano la conseguenza di qualche cambiamento, improvviso o catastrofico, o che i rifugiati possano essere, per se, un problema per i paesi di accoglienza, si potrebbe invece, tornando alla proposta di Bade (1994), vedere le migrazioni come un aspetto della condizione umana ${ }^{16}$. Quindi, periodi sedentari e migratori si alternerebbero in relazione a tempi, soggetti e luoghi; segnerebbero i ritmi e il funzionamento dei sistemi politici, dell'economia e

\footnotetext{
${ }^{15}$ Sul tema della trasposizione o trasformazione delle classificazioni amministrative e burocratiche a categorie analitiche si veda Zetter (1991).

${ }^{16}$ La migrazione è stata sempre un tema di riflessione da parte di differenti autori e in differenti prospettive, anche fuori dall'ambito dei 'refugees studies' (si veda: Arendt, 2006; Heller, Feher, 1988, p. 90; Marrus, 1985; Mauss, 1969, p. 573-639; Moore, 1989; Zolberg, 1983).
} 
dell'ambiente, sia a livello locale che globale; indicherebbero anche equilibri o squilibri dei poteri, locali e globali. $\mathrm{E}^{\prime}$ in questo contesto che sorgono le migrazioni non come un effetto, ma come una reazione prospettica, ossia come una modalità di manutenzione o ricostruzione del quotidiano. In questo senso, invece di utilizzare le categorie rifugiati o sfollati per indicare il fallimento, la violenza, la vulnerabilità o la dis-integrazione - con il rischio di renderli attori passivi -, si potrebbe sottolineare la dimensione attiva, la capacità di fare delle scelte, nonostante le limitazioni materiali e politiche. I rifugiati, in sfondo, sono persone normali che vivono una circostanza specifica. Questa circostanza non toglie la loro dignità, né li inserisce in una situazione anomala.

L'osservazione di Greta Uehling (1998) va in questo senso: "il termine rifugiato è diventato sinonimo di violazione dei diritti umani. Ma se ci soffermiamo sulla nozione di violazione, non saremo in grado di riconoscere i mezzi con cui i rifugiati costruiscono attivamente il loro mondo" (Uehling, 1998, p. 124, traduzione nostra). Questa sarebbe una sfida per le scienze sociali, ma anche per chi gestisce crisi umanitarie.

\section{Considerazioni finali}

Gli studi sulla mobilità umana tendono a rievocare l'idea dell'appartenenza ad un luogo - patria, territorio, nazione - che sarebbe alla base dell'idea di identità e ordine. La mobilità è stata associata, soprattutto per quanto riguarda le migrazioni forzate (i rifugiati), al cambiamento di tale ordine. Da ciò deriva anche l'associazione del rifugiato all'idea dello sradicato (perdita dell' appartenenza) e anche ad una minaccia per l'ordine e per la normalità del paese di destinazione. Questa visione è molto presente, principalmente nella letteratura sulla mobilità a partire dalla Seconda Guerra Mondiale, come è già stato sottolineato (Malkki, 1985). Tale prospettiva è passata per un progressivo ridimensionamento nella misura in cui ci si è concentrati sulla protezione e sui diritti dei rifugiati. In entrambe le prospettive, tuttavia, la migrazione è sempre stata vista come un problema che richiederebbe un intervento e una soluzione: un problema sociale, economico, politico e, negli ultimi anni, un problema di sicurezza; oltre ad essere, molto spesso, un problema umanitario.

Eppure la mobilità, anche quella forzata, come abbiamo visto, può essere interpretata come un modo per riordinare la vita quotidiana da parte di individui o gruppi umani. A volte perché la quotidianità è interrotta bruscamente, in altri casi perché le risorse disponibili sono scarse. In questo senso, invece di essere interpretate come problemi, le migrazioni potrebbero essere viste come modalità per affrontare i problemi, come una forma di azione. Hans Joas (1996) è particolarmente insistente nel mettere in evidenza la nozione di creatività nel concetto di azione, ossia, il modo attraverso il quale un individuo deve restaurare la quotidianità quando essa viene interrotta. Le 
modalità per le quali viene effettuata questa restaurazione sfuggono alla logica dei mezzi e dei fini: I'azione non si concretizza nello stabilire previamente un fine, indipendentemente dal contesto, per poi utilizzare i mezzi necessari per raggiungerlo. Questa sarebbe una visione strumentale dell'azione umana. In realtà, è nel contesto dell'azione, in una situazione concreta, che i fini sono definiti (Joas, 1996).

Di fronte all'interruzione o alla gravità dell'interruzione della vita quotidiana, gli individui scelgono tra le varie possibilità quelle che, al momento e in base alle loro circostanze, sono propizie per far continuare la vita, nonostante l'incertezza, la quasi totale rottura emotiva e l'estrema violenza. In questo caso, i fini emergono dal contesto della stessa azione. Quindi, la migrazione può essere interpretata come una forma residua di azione, il che sposta il tema della migrazione sulle relazioni e sulle interazioni sociali: come individui e gruppi umani interagiscono per dare un senso alle loro vite e, in questo modo, per ricostruire il loro quotidiano. Da qui l'importanza di non isolare la categoria rifugiato dal contesto in cui la migrazione forzata viene prodotta.

Questa prospettiva, come abbiamo visto, non impedisce l'elaborazione di categorie politiche e burocratiche ai fini della gestione delle crisi. Ha, tuttavia, il vantaggio di circoscrivere l'uso di queste categorie al di fuori degli scopi per cui sono state create, valutarne criticamente il funzionamento e annullare implicitamente la loro neutralità assiologica ed epistemologica. Inoltre, questa prospettiva aiuta a mettere in luce nella riflessione e nella discussione accademica aspetti della migrazione e dell'esperienza della mobilità che sfuggono al controllo ed alle classificazioni ufficiali prodotte nel contesto della gestione delle crisi umanitarie. In aggiunta, aiuterebbe anche ad ampliare il dibattito interdisciplinare sulle migrazioni e a uscire dal circolo vizioso nel quale si cade frequentemente ritornando sugli stessi temi e riproducendo le stesse categorie. Promuoverebbe, altresì, una relazione più fruttuosa tra agenzie internazionali, ONG e governi nazionali e locali: non si tratterebbe di sottomissione o scontro, e neanche di mera collaborazione, ma di promuovere la riflessione congiunta, con sufficiente spazio per la critica e la consapevolezza dei propri limiti. Aiuterà, infine, a considerare che la migrazione è un fenomeno umano e che anche i suoi protagonisti, i migranti, posseggono le loro opinioni e prospettive, e riflettono sulla mobilità. Considerare quello che pensano e fanno può aiutare a catturare aspetti della migrazione che stanno al di là del controllo burocratico e amministrativo.

\section{Riferimenti bibliografici}

ADEY, Peter. If mobility is everything then it is nothing: towards a relational politics of (im) mobilities. Mobilities, v. 1, n. 1, p. 75-94, 2006. 
AUERBACH, Jess. Flowing into the state: Returning refugee youth and citizenship in Angola. Working paper series no 68, December, Refugee Studies Centre Oxford Department of International Development, University of Oxford, 2010.

ARENDT, Hannah. Los origines del totalitarismo. Madrid: Alianza 2006.

BADE, Klaus. Homo Migrans Wanderungen aus und nach Deutschland: Erfahrungen und Fragen. Stuttgarter Vorträge zur Zeitgeschichte, 1994.

BAKEWELL, Oliver. Repatriation and Self-Settled Refugees in Zambia: Bringing solutions to the wrong problems. Journal of Refugee Studies, v. 13, n. 4, p. 356373, 2000.

BAKEWELL, Oliver. Research beyond the categories: the importance of policy irrelevant research into forced migration. Journal of Refugee Studies, v. 21, n. 4, p. 432-453, 2008.

CERTEAU, Michel. L'invention du quotidien. Arts de faire. Paris: Union Générale des Éditions, 1980.

CIRTAUTAS, Kazys C. The Refugee. A psychological study. Boston: Meador Publishing Company, 1957.

COHEN, Roberta. Response to Hathaway. Journal of Refugee Studies, v. 20, n. 3, p. 370-376, 2007.

DEWIND, Josh. Response to Hathaway. Journal of Refugee Studies, v. 20, n. 3, p. 381-385, 2007.

DILLON, Michael. Sovereignty and governmentality: From the problematics of the" New World Order" to the ethical problematic of the world order. Alternatives, v. 20, n. 3, p. 323-368, 1995.

FECHTER, Anne-Meike. Living in a bubble: Expatriates transnational spaces. In: VERET, Amit. Going first class? New approaches to privileged travel and movement. Oxford: Berghahn, 2007, p. 33-52.

FRELLO, Birgitta. Towards a discursive analytics of movement: on the making and unmaking of movement as an object of knowledge. Mobilities, v. 3, n. 1, p. 2550, 2008.

GATTI, Emanuele. Defining the expat: The case of high-skilled migrants in Brussels. Brussels Studies, v. 28, p. 1-15, 2009.

GILES-VERNICK, Tamara. We wander like birds: Migration, indigeneity, and the fabrication of frontiers in the Sangha River basin of Equatorial Africa. Environmental history, v., n. 2, p. 168-197, 1999.

GLICK SCHILLER, Nina; SALAZAR, Noel B. Regimes of mobility across the globe. Journal of Ethnic and Migration Studies, v. 39, n. 2, p. 183-200, 2013.

HABERMAS, Jurgen. Citizenship and National Identity: some reflections on the future of Europe. In: BEINER, Ronald (ed.). Theorizing Citizenship. Albany: Sunny Press, 1995, p. 255-282.

HADDAD, Emma. The refugee in international society: between sovereigns. Cambridge: Cambridge University Press, 2003. 
HANSEN, Art; TAVARES, David. Why Angolan Soldiers Worry about Demobilisation and Reintegration. In: BLACK, Richard; KOSER, Khalid (eds.). The End of The Refugee Cycle? Refugee Repatriation and Reconstruction. New York: Oxford Berghahn, 1999, p. 198-209.

HATHAWAY, James C. Forced Migration Studies: Could We Agree Just to "Date"? Journal of Refugee Studies, v. 20, n. 3, p. 349-369, 2007.

HELLER, Agnes; FEHER, Ferenc. The Postmodern Political Condition. New York: Columbia University Press, 1988.

HOLERT, Tom; TERKESSIDIS, Mark. Was bedeutet Mobilität? In: Projekt Migration. Köln: DuMont, 2005, p. 98-107.

INGLÊS, Paulo. El malestar en el postconflicto: memorias de la guerra y de la paz en Angola. Pueblos, Primero trimestre, n. 60, p. 12-15, 2014.

INGLÊS, Paulo. Avaliação do projeto "Reintegração dos angolanos expulsos da RDC". Caritas de Angola, não publicado, 2012.

INHETVEEN, Katharina. Die politische Ordnung des Flüchtlingslagers Akteure - Macht - Organisation. Eine Ethnographie im Südlichen Afrika, Bielefeld: transcript, 2010.

INSTITUTO NACIONAL DE ESTATÍSTICA. Caracterização da população deslocada e dos campos de receção. Relatório final. Luanda: MINARS (Ministério da Assistência e Reinserção Social) / UNICEF,1993.

JOAS, Hans. The Creativity of Action. Chicago: University of Chicago Press, 1996.

JÓNSSON, Gunner. Non-migrant, sedentary, immobile, or "left behind"? Reflections on the absence of migration. IMI Working Papers, v. 39. Oxford: International Migration Institute, 2011.

KAUN, Alexandra. When the Displaced Return: Challenges to Reintegration in Angola. Geneva: UNHCR, 2008.

KHOSRAVI, Shahram. The "illegal"traveller: an auto-ethnography of borders. Social anthropology, v. 15, n. 3, p. 321-334, 2007.

LANDAU, Loren B. Can We Talk and Is Anybody Listening? Reflections on IASFM 10,"Talking Across Borders: New Dialogues in Forced Migration". Journal of Refugee Studies, v. 20, n. 3, p. 335-348, 2007.

LUBKEMANN, Stephen C. Involuntary immobility: on a theoretical invisibility in forced migration studies. Journal of Refugee Studies, v. 21, n. 4, p. 454-475, 2008.

MALKKI, Liisa. The Origin of a Device of Power: The Refugee Camp in Post-War Europe. unpublished MS. Anthropology Department, Hanard University, 1985.

MALKKI, Liisa. Purity and Exile: Violence, Memory and National Cosmology Among Hutu Refugees in Tanzania. Chicago: University of Chicago Press, 1995.

MALKKI, Liisa. National geographic: the rooting of peoples and the territorialization of national identity among scholars and refugees. Cultural anthropology, v. 7, n. 1, p. 24-44, 1992.

MARRUS, Michael. The Unwanted: European Refugees in the Twentieth Century. New York: Oxford University Press, 1985. 
MAUSS, Marcel. La nation. In: Oeuvres. v. 3. Paris: Les Éditions du Minuit, 1969.

MOORE, Sally Falk. The production of cultural pluralism as a process. Public culture, v. 1, n. 2, p. 26-48, 1989.

NETO, Maria da Conceição. Ideologias, contradições e mistificações da colonização de Angola no século XX. Lusotopie, n. 4, p. 327-359, 1997.

RICHMOND, Anthony. Reactive Migration: Sociological Perspectives On Refugee Movements. Journal of Refugee Studies, v. 6, n. 1, p. 7-24, 1993.

SIMPSON, John Hope. The Refugee Question. London: Oxford University Press, 1939.

SOYSAL, Yasemin. Nuhoglu. Citizenship and identity: living in diasporas in post-war Europe? Ethnic and racial studies, v. 23, n. 1, p. 1-15, 2000.

UEHLING, Greta. Is there "refuge" in the refugee category? Power, Ethics and Human Rights: Anthropological studies of refugee research and action, Rowman and Littlefield Publishers: Maryland, p. 123-144, 1998.

VANSINA, Jan. Kingdoms of the Savanna. Madison. Wisconsin: University of Wisconsin Press, 1966.

VON OPPEN, Achim. Terms of Trade and Terms of Trust: The History and Contexts of Pre-colonial Market Production around the Upper Zambezi and Kasai. Studien zur afrikanischen Geschichte 6, Münster \& Hamburg: LIT, 1994.

ZETTER, Roger. Labelling refugees: forming and transforming a bureaucratic identity. Journal of refugee studies, v. 4, n. 1, p. 39-62, 1991.

ZOLBERG, Aristide. The Formation of New States as a Refugee-Generating Process. In: LOESCHER, Gilburt; SCANLAN, John (eds.). The Global Refugee Problem. Beverly Hills, California: Sage, 1983, p. 24-38.

Articolo ricevuto il 15.08.2018

Accettato per la pubblicazione 03.11.2018

Received for publication in August 15 $5^{\text {th }}, 2018$

Accepted for publication in November 03 ${ }^{\text {rd }}, 2018$

ISSN impresso 1980-8585

ISSN eletrônico 2237-9843

http://dx.doi.org/10.1590/1980-85852503880005406 